\title{
MÓDULO EDUCATIVO PARA ENSINO EM ENGENHARIA QUÍMICA: SISTEMA DE TANQUES EM SÉRIE E REATOR BIOQUÍMICO
}

\author{
K. A. SILVA ${ }^{1}$, I. D. C. MONTANO ${ }^{1}$, C. A. G. SUAREZ ${ }^{1}$ \\ ${ }^{1}$ Universidade Federal de Goiás, Instituto de Química, Curso de Engenharia Química \\ E-mail para contato: carlogalen21@gmail.com
}

\begin{abstract}
RESUMO - A utilização de programas de computador no ensino da engenharia química não só prepara melhor o estudante para as diferentes ferramentas que utilizarão no exercício da profissão, como também possibilita um entendimento mais profundo dos diferentes sistemas de uma indústria química. Dessa forma, este trabalho teve como objetivo desenvolver um módulo educativo que permita aos estudantes simular um sistema de tanques em série em regime contínuo, um sistema de tanques em série em regime semi-contínuo, um reator de hidrólise enzimática e uma biorreator de crescimento microbiano.
\end{abstract}

\section{INTRODUÇÃO}

A evolução tecnológica na área da informática possibilitou o surgimento de programas de simulação que permitem a predição do comportamento dos sistemas em diferentes condições (SCHULTZ et al., 2014). As vantagens que esses simuladores trouxeram para as indústrias químicas são inúmeras e vão desde a redução de custos com testes experimentais, que, além de onerosos, podem ser demorados e demandam recurso humano, até a liberação do engenheiro de projeto para se concentrar em detalhes mais importantes. É imprescindível, por tanto, que o ensino de engenharia química permita que os estudantes desenvolvam suas habilidades em informática, garantindo um melhor preparo para enfrentar os desafios que encontrão na sua carreira profissional (LEITE et al., 2014).

Ademais, a inclusão de programas de simulação durante a graduação facilita a compreensão do comportamento dos sistemas, permitindo que os estudantes visualizem de forma rápida e clara os efeitos que mudanças nas variáveis causam no desempenho dos processos (LEITE et al., 2014).

Os simuladores preveem o comportamento dos sistemas pela resolução de modelos matemáticos, que são constituídos por equações que descrevem a variável de saída em função das de entrada. Pode-se tratar de um sistema de equações algébricas quando se assume estado estacionário ou de um sistema de equações diferenciais quando o processo está em estado transiente. Normalmente, utilizam-se métodos numéricos como os métodos interativos e o de Runge-Kutta na resolução desses sistemas de equações (SCHULTZ et al., 2014). 


\section{MODELO}

O equacionamento dos sistemas estudados baseou-se apenas no princípio da conservação da massa, não tendo sido considerado variações de temperatura.

\subsection{Sistema de tanques em série}

Utilizaram-se dois diferentes sistemas de tanques em série, um operando em regime contínuo e o outro em regime semi-contínuo, conforme ilustrado pela Figura 1.

Figura 1: Sistema de tanques em série: (a) operação contínua; (b) operação semi-contínua.

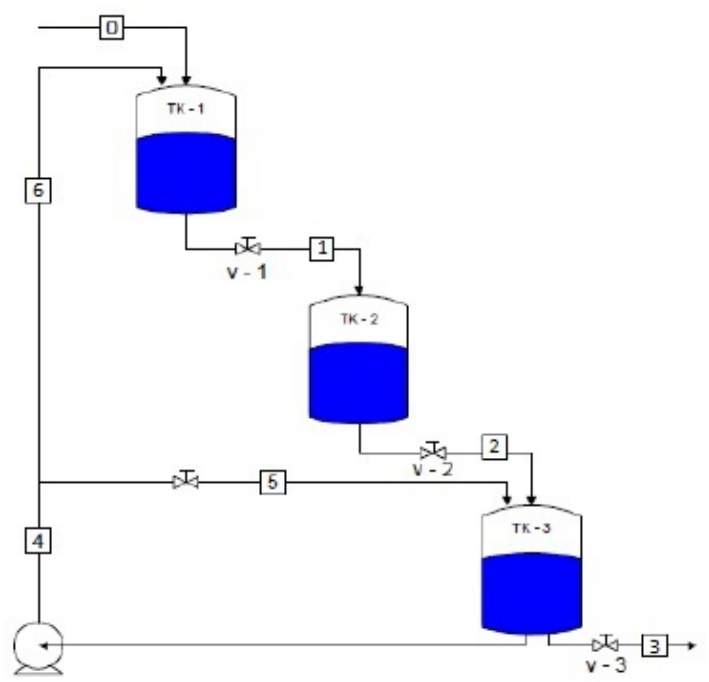

(a)

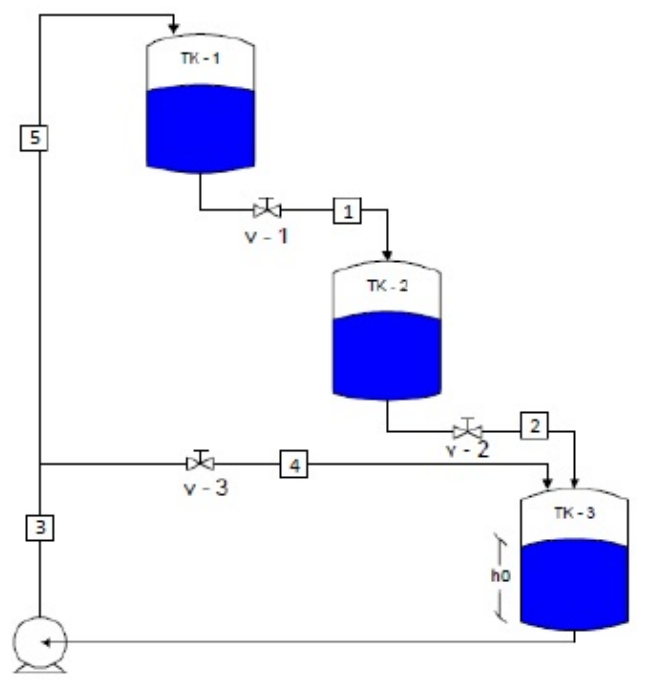

(b)

Assumiu-se para ambos os sistemas operação transiente, fluido incompressível, mistura perfeita e seção transversal dos tanques constantes. O sistema de equações ( 1 até 9 ) representa a operação contínua.

$$
\begin{aligned}
& F_{1}=C v_{1} * \sqrt{H_{1}} \\
& F_{2}=C v_{2} * \sqrt{H_{2}} \\
& F_{3}=C v_{3} * \sqrt{H_{3}} \\
& F_{4}=C v_{4} * \sqrt{H_{3}} \\
& F_{5}=R * F_{4} \\
& F_{6}=(1-R)^{*} F_{4} \\
& \frac{d H_{1}}{d t}=\frac{F_{0}+F_{6}-F_{1}}{A_{1}}
\end{aligned}
$$




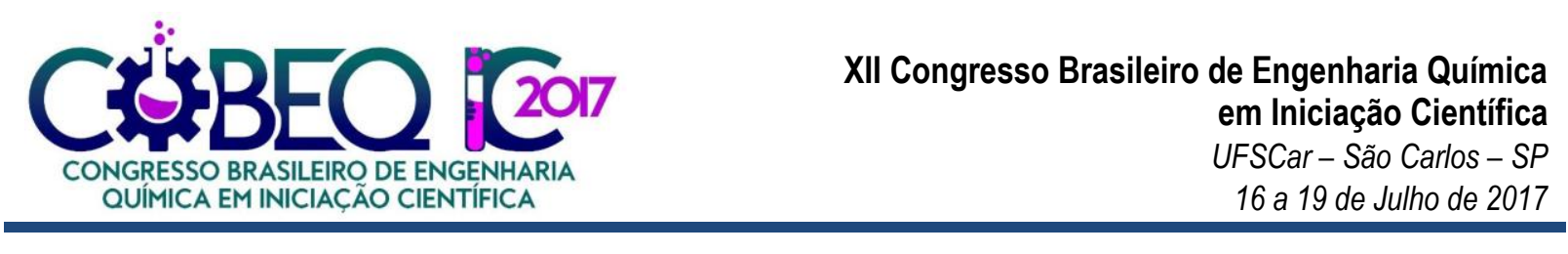

$$
\begin{aligned}
& \frac{d H_{2}}{d t}=\frac{F_{1}-F_{2}}{A_{2}} \\
& \frac{d H_{3}}{d t}=\frac{F_{2}+F_{5}-F_{3}-F_{4}}{A_{3}}
\end{aligned}
$$

A Tabela 1 mostra as variáveis conhecidas e os parâmetros, que devem ser informados pelo usuário e as variáveis desconhecidas, que serão determinadas pela resolução do sistema de equações.

Tabela 1: Variáveis conhecidas e desconhecidas e parâmetros dos sistemas de tanques em série

\begin{tabular}{|c|c|c|}
\hline & $\begin{array}{c}\text { Sistema } \\
\text { contínuo }\end{array}$ & $\begin{array}{c}\text { Sistema semi- } \\
\text { contínuo }\end{array}$ \\
\hline \hline $\begin{array}{c}\text { Variáveis } \\
\text { conhecidas }\end{array}$ & $\begin{array}{c}\mathrm{F}_{0}, \mathrm{~A}_{1}, \mathrm{~A}_{2}, \mathrm{~A}_{3}, \\
\mathrm{R}\end{array}$ & $\mathrm{A}_{1}, \mathrm{~A}_{2}, \mathrm{~A}_{3}, \mathrm{R}$ \\
\hline $\begin{array}{c}\text { Variáveis } \\
\text { desconhecidas }\end{array}$ & $\begin{array}{c}\mathrm{F}_{1}, \mathrm{~F}_{2}, \mathrm{~F}_{3}, \mathrm{~F}_{4}, \\
\mathrm{~F}_{5}, \mathrm{~F}_{6}, \mathrm{H}_{1}, \mathrm{H}_{2}, \\
\mathrm{H}_{3}\end{array}$ & $\begin{array}{c}\mathrm{F}_{1}, \mathrm{~F}_{2}, \mathrm{~F}_{3}, \mathrm{~F}_{4}, \mathrm{~F}_{5}, \\
\mathrm{H}_{1}, \mathrm{H}_{2}, \mathrm{H}_{3}\end{array}$ \\
\hline Parâmetros & $\begin{array}{c}\mathrm{Cv}_{1}, \mathrm{Cv}_{2}, \mathrm{Cv}_{3}, \\
\mathrm{Cv}_{4}\end{array}$ & $\mathrm{Cv}_{1}, \mathrm{Cv}_{2}, \mathrm{Cv}_{3}$ \\
\hline
\end{tabular}

O grau de liberdade tanto da operação contínua quanto da semi-contínuo é zero. Portanto, trata-se de sistemas consistentes e determinados. Uma vez que há equações diferenciais, também é necessário especificar as condições iniciais $\mathrm{H}_{1}(0), \mathrm{H}_{2}(0)$ e $\mathrm{H}_{3}(0)$.

\subsection{Biorreator para crescimento microbiano}

Optou-se por modelar um biorreator para crescimento de Escherichia coli, que utiliza glicose como substrato. Foi assumido que o sistema opere em batelada e em regime transiente e que se trata de um reator perfeitamente agitado de volume constante. Ademais, utilizou-se a equação de Monod para representar a taxa de reação. $O$ sistema de equações $(10$ e 11) representa o processo estudado.

$$
\begin{aligned}
& \frac{d X}{d t}=X * \frac{\mu_{\max } * S}{K_{S}+S} \\
& \frac{d S}{d t}=-\frac{\mu_{\max }}{Y} * \frac{X * S}{K_{S}+S}
\end{aligned}
$$

Os parâmetros utilizados foram os descritos por Rosa et al. (2014): $\mu_{\max }=0,619 \mathrm{~h}^{-1}$, $\mathrm{K}_{\mathrm{s}}=0,103 \mathrm{~g} / \mathrm{l}$ e $\mathrm{Y}=0,5 \mathrm{~g}$ células/g substrato. $\mathrm{O}$ grau de liberdade do sistema é zero, sendo o sistema consistente e determinado. É necessário especificar as condições iniciais $X(0)$ e $S(0)$.

\section{IMPLEMENTAÇÃO DO MÓDULO EDUCATIVO}

O programa foi desenvolvido no Visual Basic (VBA), que é uma plataforma de programação inserida no Microsoft Office Excel. 
Primeiramente, foi criada a interface de comunicação com o usuário. Depois, desenvolveu-se o código de comando dentro do qual foram incorporados os modelos matemáticos dos sistemas.

Utilizou-se o método de Runge - Kutta de $4^{\circ}$ ordem para resolver os sistemas de equações ordinárias. Assim, o usuário deverá fornecer o passo (h) e o ponto final ( $t_{\text {final }}(h)$ ).

Por fim, verificaram-se os resultados gerados pelo módulo educativo com os de outro programa computacional com o objetivo de constatar se o método numérico utilizado funciona perfeitamente.

\section{RESULTADOS E DISCUSSÃO}

A interface do programa foi desenvolvida buscando facilitar a sua utilização. Assim, o usuário pode escolher apenas com um clique o sistema que desejar. Ademais, há um botão de ajuda, o qual fornece informações sobre o funcionamento do programa. A Figura 2 apresentam a interface do programa.

Figura 2: Interfaces iniciais: (a) módulo educativo; (b) Interface do sistema contínuo

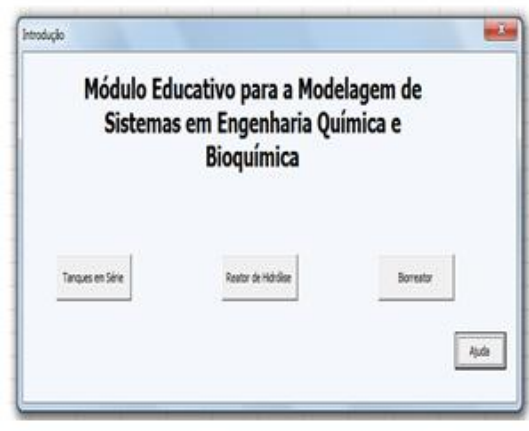

(a)

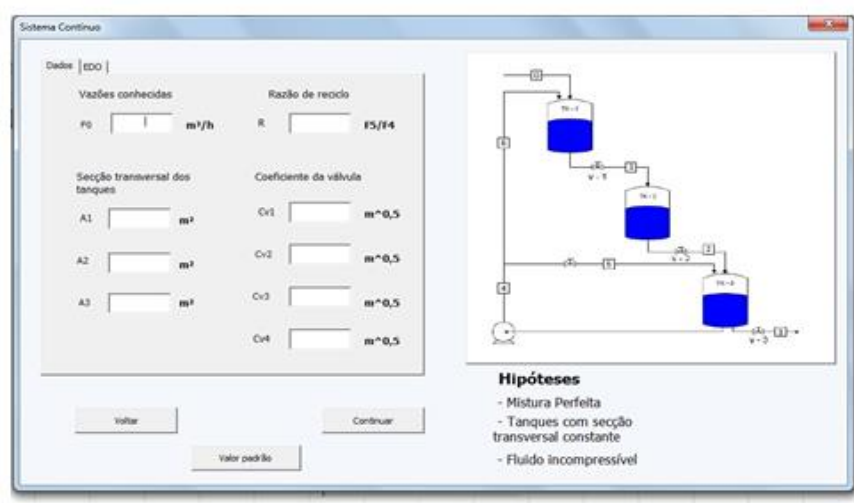

(b)

Em cada sistema, o usuário pode optar por preencher os campos dos dados com valores pré-determinados no programa clicando no botão Valor padrão.

Cada sistema do módulo educativo foi testado com dois grupos diferentes de dados de entrada, sendo o resultado obtido comparado com o fornecido por outro programa.

Conforme pode ser observado nas Figuras 3 e 4, o perfil do nível dos tanques gerado pelo módulo educativo tanto para os tanque em série em regime contínuo quanto para o semicontínuo se assemelha ao obtido no programa utilizado como referência. 
Figura 3: Gráficos do sistema de tanques em série em regime contínuo: módulo educativo $\mathrm{h}=0,5 ;(\mathrm{b})$ programa de referência

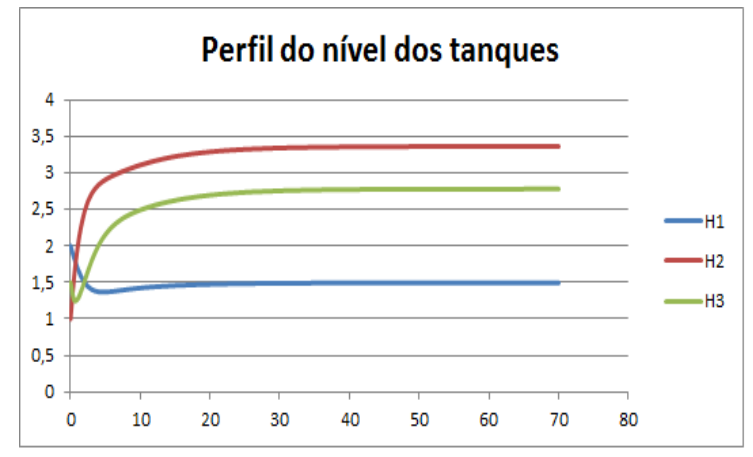

(a)

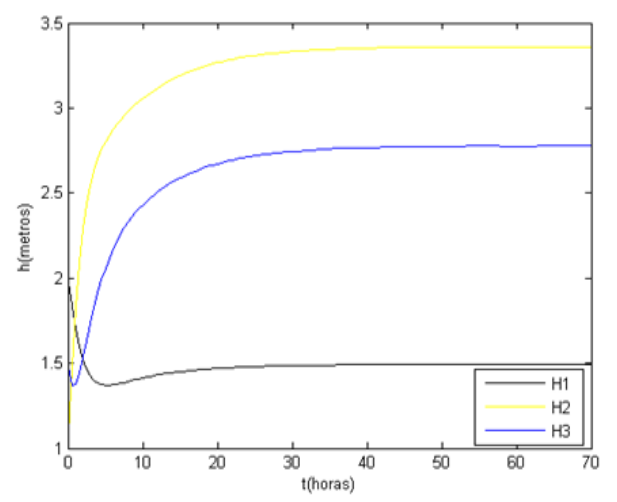

(b)

Figura 4: Gráficos do sistema de tanques em série em regime semi-contínuo: (a) módulo educativo $\mathrm{h}=0,1$; (b) programa de referência

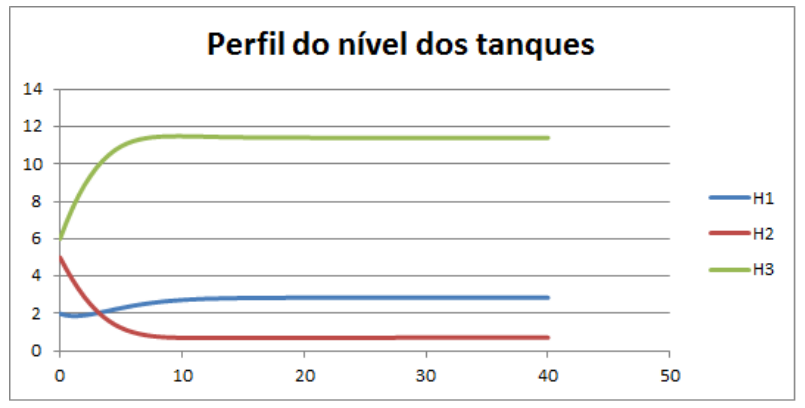

(a)

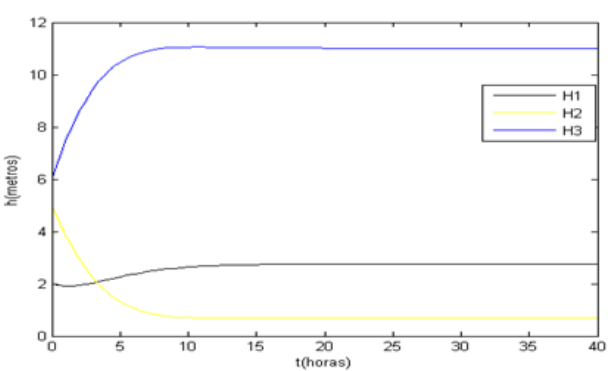

(b)

A simulação do sistema em regime contínuo apresentou o melhor desempenho, sendo o maior erro encontrado igual a $0,058 \%$ para o tanque 3 na primeira simulação. Já no regime semi-contínuo, o erro obtido foi um pouco maior de $1,3 \%$ na simulação 1 e $3,5 \%$ na simulação 2, como mostrado na Tabela 2.

Tabela 2 - Valores obtidos para tfinal sistema de tanques em série

\begin{tabular}{|c|c|c|c|c|c|c|c|c|c|}
\hline \multicolumn{10}{|c|}{ Tanques em série: regime contínuo } \\
\hline & \multicolumn{3}{|c|}{$\mathrm{H}_{1}$} & \multicolumn{3}{|c|}{$\mathrm{H}_{2}$} & \multicolumn{3}{|c|}{$\mathrm{H}_{3}$} \\
\hline $\begin{array}{l}\text { Simul } \\
\text { ação }\end{array}$ & $\mathrm{ME}$ & PR & Erro\% & $\mathrm{ME}$ & PR & Erro\% & ME & PR & Erro\% \\
\hline 1 & 25,000 & 25,000 & 0,000 & 24,985 & 24,982 & 0,010 & 24,887 & 24,873 & 0,058 \\
\hline 2 & 1,494 & 1,494 & 0,000 & 3,361 & 3,361 & 0,003 & 2,778 & 2,778 & 0,004 \\
\hline \multicolumn{10}{|c|}{ Tanques em série: regime contínuo } \\
\hline & \multicolumn{3}{|c|}{$\mathrm{H}_{1}$} & \multicolumn{3}{|c|}{$\mathrm{H}_{2}$} & \multicolumn{3}{|c|}{$\mathrm{H}_{3}$} \\
\hline & ME & PR & Erro\% & $\mathrm{ME}$ & PR & Erro\% & $\mathrm{ME}$ & PR & Erro\% \\
\hline 1 & 1,675 & 1,653 & 1,337 & 6,701 & 6,612 & 1,349 & 0,745 & 0,735 & 1,348 \\
\hline 2 & 2,849 & 2,753 & 3,494 & 0,712 & 0,688 & 3,487 & 11,398 & 11,013 & 3,491 \\
\hline
\end{tabular}

ME: módulo educativo; PR: programa de referência. 
Ruggiero e Lopes (1996) apontaram que a desvantagem do método de Runge-Kutta reside na impossibilidade de se fazer uma estimativa simples para o erro o que dificulta a escolha do passo $\mathrm{h}$. Considerando esse fator, foi investigado o efeito no erro ao reduzir o passo utilizado. Essa análise não foi feita para os tanques em série em regime contínuo por ter sido obtido erros pequenos na simulação com passo de 1 (simulação 1) e de 0,5 (simulação 2).

\section{NOMENCLATURA}

F1 - vazão volumétrica que sai do tanque $1\left(\mathrm{~m}^{3} / \mathrm{h}\right) ; \mathrm{Cv}_{\mathrm{i}}$ - constante característica da válvula $1\left(\mathrm{~m}^{5 / 2} / \mathrm{h}\right) ; \mathrm{H}_{\mathrm{i}}$ - altura do tanque $1(\mathrm{~m}) ; \mathrm{F}_{2}$ - vazão volumétrica que sai do tanque 2 $\left(\mathrm{m}^{3} / \mathrm{h}\right) ; \mathrm{F}_{3}$ - vazão volumétrica que sai do tanque $3\left(\mathrm{~m}^{3} / \mathrm{h}\right) ; \mathrm{F}_{4}$ - vazão volumétrica de recirculação total $\left(\mathrm{m}^{3} / \mathrm{h}\right) ; \mathrm{F}_{5}$ - vazão volumétrica de recirculação do tanque $3\left(\mathrm{~m}^{3} / \mathrm{h}\right) ; \mathrm{R}$ - razão de recirculação para o tanque $3 ; F_{6}$ - vazão volumétrica de recirculação do tanque $1\left(\mathrm{~m}^{3} / \mathrm{h}\right) ; \mathrm{t}$ tempo (h); $\mathrm{F}_{0}$ - vazão de alimentação $\left(\mathrm{m}^{3} / \mathrm{h}\right) ; \mathrm{A}_{\mathrm{i}}$ - seção transversal do tanque $1 ; \mathrm{X}$ concentração de Escherichia coli (g/l); $\mu_{\max }$ - crescimento específico máximo (1/h); S concentração de substrato (g/l); $\mathrm{K}_{\mathrm{S}}$ - constante de meia saturação do substrato $\mathrm{S}(\mathrm{g} / \mathrm{l})$; Y rendimento celular (g células/g substrato).

\section{CONCLUSÃO}

O módulo educativo desenvolvido neste trabalho é uma ferramenta que possibilita conhecer, analisar e simular a dinâmica para quatro sistemas diferentes, obtendo resultados satisfatórios quando comparados com outro tipo de software nas mesmas condições.

A performance de cada modelo foi analisado com dois grupos de dados de entrada. Todos os quatro sistemas apresentaram desempenho satisfatório com erros menores que $1 \%$. No entanto, isso foi alcançado utilizando passos de diferentes magnitudes para cada sistema.

O sistema de tanques em série em regime contínuo pode ser utilizado com passos da ordem de 1 unidade. O sistema de tanques em série em regime semi-contínuo, por sua vez, apresentou desempenho aceitável com passo da ordem de 0,1 . Já o sistema de hidrólise enzimática deve ser usado com passo da ordem de 0,01 , enquanto o sistema de crescimento microbiano exige passo ainda menor, da ordem de 0,001 .

Finalmente o uso do programa permite a resolução de problemas típicos da engenharia química e/ou bioquímica, usando o método matemático de Runge-Kutta de 4ta ordem de uma forma fácil e intuitiva, o que viabiliza a aceitação do programa entre os alunos de engenharia química na analise da dinâmica e simulação de processos.

\section{REFERÊNCIAS}

LEITE, J. P.; Beltrão M.J.C.; CHEBAR, I. E.; RAMIREZ, N. I. B. Avaliação Preliminar do Impacto de Softwares de Simulação no Ensino das Engenharias Química e de Petróleo. Engevista, v. 16, p.28-40, 2014.

ROSA, M. F.; MONTANO, I. D. C.; HORTA, A.C. L. Definição de um Modelo não Estruturado para Simulação de Cultivos em Batelada de E. coli Recombinante com Vários Substratos. In: JORNADA EM ENGENHARIA QUÍMICA, 19., 2014, Uberlândia.

RUGGIERO, M. A. G.; LOPES, V. L. R. Cálculo Numérico: aspectos teóricos e computacionais. 2. ed. São Paulo: Makron Books, 1996.

SCHULTZ, G.; LAUER, J.; LOPES, J.M.; RANGEL, R.S.; SIMONELLI, G. Modelagem e Simulação dos Reatores Químicos BR e PFR no EMSI e GNU Octave. Enciclopédia Biosfera, v. 10, p.3736-3757, 2014. 